Euskal ikerketen aldizkaria | Revue d'études basques |

Revista de estudios vascos | Basque studies review

$16 \mid 2012$

Numéro XVI

\title{
Les éditions des contes basques : les textes sources et les éditions contemporaines
}

\author{
Natalia M. Zaïka
}

\section{OpenEdition \\ Journals}

Édition électronique

URL : http://journals.openedition.org/lapurdum/2396

DOI : 10.4000/lapurdum.2396

ISSN : 1965-0655

\section{Éditeur}

IKER

\section{Édition imprimée}

Date de publication : 1 octobre 2012

Pagination : 153-179

ISSN : 1273-3830

Référence électronique

Natalia M. Zaïka, «Les éditions des contes basques : les textes sources et les éditions

contemporaines », Lapurdum [En ligne], 16 | 2012, mis en ligne le 03 décembre 2014, consulté le 06 mai 2019. URL : http://journals.openedition.org/lapurdum/2396 ; DOI : 10.4000/lapurdum.2396 


\title{
Les éditions des contes basques: les textes sources et les éditions contemporaines
}

\author{
Natalia M. ZAÏKA \\ L'Institut de Recherches Linguistiques de l'Académie des Sciences de la \\ Russie / L'Université d'Etat de Saint-Pétersbourg
}

\section{Introduction}

Dans notre article ${ }^{1}$, nous étudierons le fonctionnement des contes basques dans les recueils du XX ${ }^{\text {ème }}$ siècle, dont la plupart sont des livres de vulgarisation et non pas des travaux scientifiques. Nous analyserons plusieurs recueils de contes basques et les transformations que ces contes ont subies par rapport à la version originale.

A la seconde moitié du XXème siècle, on peut observer partout en Europe, en France et au Pays basque un retour vers les sources, et le folklore commence à nouveau par être apprécié.

Comment les textes folkloriques basques ont été utilisés ? Quels types de récits folkloriques sont plus répandus dans les livres de «vulgarisation », les contes merveilleux ou les autres contes et légendes plus typiques au Pays basque?

Bien que dans notre article il s'agisse des recueils contemporains, nous aborderons tout d'abord les problèmes du fonctionnement des textes basques dans les premières éditions.

La commercialisation du folklore basque est un processus normal et contemporain qui est caractéristique de beaucoup d'autres pays. Une partie de ce processus consiste à publier des livres sur les sujets folkloriques, qui peuvent s'éloigner de l'original. Dans certains recueils, qui ont très peu en commun avec le folklore basque, il s'agit d'histoires de vie, qui se passent au Pays basque ${ }^{2}$. Nous ne les analyserons pas dans notre article, puisqu'ils ne sont pas dérivés

1.- Notre article est fondé sur un chapitre de notre thèse « Approche textologique et comparative du conte traditionnel basque dans les versions bilingues de 1873 à 1942 (W. Webster, J.-F. Cerquand, J. Barbier, R. M. de Azkue) » sous la direction de Mme Aurélie Arcocha-Scarcia, Professeur de l'Université Michel MontaigneBordeaux 3, UMR-5478 IKER (CNRS - Bordeaux 3 - UPPA) et M. Viktor S. Khrakovsky, chef de laboratoire à l'Institut de Recherches Linguistiques de l'Académie des Sciences de la Russie.

2.- Cf. également Euskal Herriko Kondairak [EHK 1986] et d'autres. 
des recueils traditionnels ${ }^{3}$.

Nous pouvons également citer un livre de Souberbieille, un recueil de contes où l'auteur utilise des motifs basques. Ainsi, l'un des contes La jeune Basquaise et le roi [Souberbieille 2005 : 34-40] est un arrangement littéraire d'un conte traditionnel qui est courant au Pays basque 4 . Un autre conte (Le Diable et le Basque [Souberbieille 2005 : 59-63]) évoque la légende du diable qui n'a pas pu apprendre la langue basque.

Les premiers folkloristes attachent beaucoup d'importance à la traduction des contes dans une langue romane et à la présentation du texte original en basque. Ainsi, Barbier dit que sans le texte basque, le livre serait « un recueil, comme il y en a tant, un peu suspect de fantaisie et toujours très banal » et que « la traduction de ces morceaux devait être à peu près littérale » [Barbier 1931 : 5]. Cependant, malgré le fait que la plupart des premiers recueils aient été bilingues, les recueils postérieurs sont presque toujours monolingues.

Il faut souligner qu'en ce qui concerne l'histoire textuelle des contes, même le texte des premiers folkloristes n'est pas toujours authentique. De plus, dès le début de la collecte, les chercheurs commencent à romantiser les contes. Ainsi, très peu de temps après la publication de la première édition de Webster, en 1878, Manuel Gorostidi écrit :

\begin{abstract}
«Las leyendas llamadas por los naturales « Lege zaharreco istoriyuac ${ }^{5}$, son trasmitidas de padres á hijos, desde la época más remota, refiriéndose, en torno del hogar, durante las largas veladas del invierno, cuando, terminadas las faenas del día, se retiran á los solitarios caseríos enclavados en medio de une naturaleza magestuosa é imponente » [Gorostidi 1878 : 177-178].
\end{abstract}

Pourtant, à l'époque où l'on recueillait les contes basques, la tradition des veillées était en train de disparaître, et tous ou presque tous les contes des collecteurs basques reconnus ont été collectés dans des conditions artificielles.

Les contes ont normalement de nombreuses variantes, ils évoluent de recueil en recueil. Par exemple, nous pouvons parler d'un conte qui s'appelle Malbrouc paru dans Basque Legends de Webster en anglais [Webster 1877 : 77-86], d'un autre conte Malbrouk, dans le recueil de Vinson Le folklore du Pays Basque en français [Vinson 1883 : 80-92], du texte de la nouvelle traduction de Basque Legends en français [Webster 2005 : 123-134], ainsi que de plusieurs textes en basque et en français dans le manuscrit de Webster [1873: 464-479] et de Vinson [1876: 77-78].

3.- Nous entendons par les recueils traditionnels les textes suivants : Euskalerriaren Yakintza. Literatura popular del País Vasco de Resurrección Maria de Azkue (1935-1947), volume 2 qui inclut les contes [Azkue 1942], Légendes \& Récits Populaires du PAYS BASQUE de Jean-François Cerquand [Cerquand 1874-1882] publiés dans le Le Bulletin de la Société des Sciences, Lettres et Arts de Pau, Basque Legends de Wentworth Webster (livres parus en 1877 et 1879 et le manuscrit du folkloriste) [Webster 1877, Webster 1879 et Webster 1873], Légendes et contes basques de Jean Barbier [Barbier 1931] et El mundo en la mente popular vasca de José Miguel de Barandiarán y colaboradores [Barandiarán et al. 1960-1962].

4.- Cf., par exemple, [Cerquand 1876 : 529-531].

5.- Les histoires de l'ancienne loi. 
Mais si dans ce cas il s'agit plus ou moins du même texte, il existe des contes qui changent radicalement au cours du temps. Parfois les auteurs mélangent les variantes des contes et en créent une image qui leur paraît plus complète. C'est le cas du conte des deux bossus qui a été pris par Julien Vinson chez Cerquand [Vinson 1883 : 14-16, Cerquand 1875 : 236-238], ainsi que d'autres contes.

La forme succincte des contes basques qui se caractérise par l'absence des enjolivements est parfois perçue comme un résultat des changements ${ }^{6}$. Par ailleurs, des traductions successives peuvent aboutir à des variantes qui s'éloignent de l'original. Ainsi, le titre Tabakiera du manuscrit de Webster en basque [Webster 1873: 354] devient Tabatière dans le recueil de Vinson en français [Vinson 1883 : 63], et il est traduit de nouveau comme Tabakontzia. [MLV 1986 : 112].

\section{Le choix des contes}

Envisageons à présent les questions liées à l'histoire textuelle des recueils. Comment se transformaient les contes basques de recueil en recueil ? Quels sont les contes choisis par les éditeurs contemporains s'agissant de contes authentiques, ces récits qui se transmettent de génération en génération? Ce sont souvent ceux des recueils classiques que l'on choisit et que l'on modifie.

Tout d'abord, il convient de mentionner les éditions modernes des recueils classiques où l'on ne mélange pas des contes collectés par les folkloristes différents.

L'édition récente des contes basques de Cerquand transcrite par Anuntzi Arana [Cerquand 1985, 1986] est l'une des meilleures. Lorthographe est moderne, les mots dialectaux sont traduits en basque unifié dans les notes de bas de page. Le transcripteur y ajoute quelques notes comparatives: Arana compare les contes de Cerquand à ceux de Barbier et indique la topographie des lieux.

Parmi d'autres éditions récentes de contes qui appartiennent à un seul auteur, nous pouvons mentionner celle d'Azkue [Azkue 1968] : le texte est publié 17 ans après la mort du savant et contient 62 contes, environ un quart des textes publiés dans le deuxième tome de son travail folklorique [Azkue 1942].

Pourtant, mis à part ce genre de recueils, il en existe beaucoup qui présentent une compilation de textes appartenant à plusieurs recueils, très souvent, modifiés. Les textes sont souvent comparés à ceux de Webster, Vinson, Barandiarán.

Il est possible de considérer les recueils de contes comme une sorte du corpus, car les compilateurs sont souvent obligés de choisir une vingtaine, une trentaine ou plus de contes parmi quelques centaines. Ainsi, ayant choisi 99 textes, l'éditeur d'un recueil explique son choix : « nous nous sommes efforcés de retenir les variantes les plus typées » [CMF 1991 : ii]. Il est intéressant que les auteurs de compilations essayent de créer de nouvelles classifications des contes.

6.- Cf. [CMF 1991 : 42] où il s'agit de Tartaro de Webster : "Le Tartaro a été traduit du basque en anglais et de là en français, d'où sa forme un peu sèche et résumée ». 
Les principes selon lesquels les auteurs choisissent les contes et les adaptent sont souvent cités :

« les contes de toujours qui viennent de tous les endroits, mais en basque, c'est cela que nous voulons recueillir dans ce livre. En effet, beaucoup de gens seront étonnés, en lisant nos contes, d'y trouver d'autres pays et des histoires d'autres cultures dans la nôtre. Par exemple, c'est le cas du conte basque intitulé La lampe magique en comparaison a l'histoire d'Aladdin; ou bien le Tartalo, le frère du Ciclope classique chez nous. Dans ce recueil, le lecteur trouvera également quelques contes des frères Grimm [...]. En plus, nous avons publié les différentes éditions des mêmes contes, puisque le lien entre les peuples et les cultures et cette abondance est le signal de ce qui s'est passé au Pays Basque » [Etxaniz $2000: 7]^{7}$.

Quelques compilateurs éclaircissent leurs raisons comme le fait San Martín :

« En choisissant, nous n'avons pas seulement regardé s'ils sont les meilleurs, mais parmi les meilleurs, sur des sujets différents, et en plus des dialectes différents, de tout le Pays basque » [Azkue 1968: 11] ${ }^{8}$.

Pourtant, les principes du choix des contes modernes ne sont pas toujours évidents. Les compilateurs qui ne les expliquent pas choisissent plutôt des recueils plus connus, se fondant sur trois ou quatre livres environ.

Nous pouvons remarquer que pour beaucoup d'éditeurs, les contes basques restent tout d'abord des contes d'Espagne ou de France, puisque les éditeurs espagnols préfèrent le recueil de Barandiarán et les Français ceux de Barbier et de Cerquand ${ }^{9}$. Ainsi, Angel Irigarai dit dans son recueil [Irigarai 1957: 121] que la plupart de ses contes ont déjà été publiés par José Miguel de Barandiarán.

Un autre éditeur de contes, Beobide'tar José Luis, signale sous le titre de son livre: « Nous devons à Azkue, Barandiarán, Estonba et à d'autres personnes qui ont ressemblé les contes ce recueil des contes $»^{10}$ [Beobide’tar 1980 : 1]. Labsence de contes du Pays basque d'Espagne dépend souvent de la tradition française qui situe les contes basques parmi les

7.- « ... betiko ipuinak, leku guztietakoak, baina eukaraz », horixe da liburu honetan bildi nahi duguna. Izan ere, bat baino gehiago harrituko da gure ipuin hauek irakurtzean beste herrialde, beste kulturetako istorioak gurean ere aurkitzean. Hor ditugu, esaterako, « Lanpara poderosa » izeneko euskal ipuina Aladinoren istoriaren ondoan ; edo Ziklope klasikoaren anaia gure artean : Tartaroa ; Grimm anaien ipuinetako hainbat istorio ere aurkituko ditu irakurleak bilduma honetan [...]. Halaber, ipuin berberaren bertsio ezberdinak ere jarri ditugu, aberastasun hori, herrien arteko eta kulturen arteko lotura eta ugaritasuna Euskal Herrian bertan ere gertatu den seinale ».

8.- « Autapenak egitean ez diogu begiratu onenak izateari bakarrik, baizik ontxoenen artean gaiez desberdiñenak eta gaiñera euskalki guztietarik zetxobait arturik, Euskalerriaren zabalera guztiokoekin ornitzearren ».

9.- Ce qui ne les empêche pas d'utiliser d'autres sources, nous parlons ici d'une tendance plutôt que d'une règle absolue.

10.- « Azkue, Barandiarán, Estonba eta beste euskal-ipuin batzailleai zor dautsegu ipuin-sorta hau ». 
contes de France. Ainsi Pierre d'Anjou publie son recueil dans la collection Contes de France, ce qui explique aussi le choix des folkloristes dont il utilise les contes.

En revanche, nous constatons que, même dans les éditions plus récentes qui sont publiées en France, les recueils d'Azkue et de Barandiarán sont plus rarement employés, ce qui peut être expliqué par le fait que leurs versions bilingues étaient basco-espagnoles.

Dans le recueil des contes populaires basques paru en France [CB 1978], on utilise les contes de Webster, Barbier, Cerquand, Vinson et Francisque Michel. Néanmoins, les recueils modernes se servent des sources espagnoles aussi bien que françaises. Xabier Etxaniz qui habite au Pays basque d'Espagne utilise dans son recueil des textes de Cerquand, Webster et Barbier, ainsi que ceux d'Azkue et Barandiarán. Etxaniz reconnaît certaines modifications dans le texte, tandis que plusieurs éditeurs ne le font pas, et pour une meilleure compréhension les contes du recueil sont traduits en basque unifié.

Le choix des contes dépend aussi du lecteur du livre. Qui sont les lecteurs potentiels des contes merveilleux basques de la fin du XIX ${ }^{\text {ème }}$ et du début du XX ${ }^{\text {ème }}$ siècle et des recueils postérieurs? Ces textes, sont-ils prédestinés à des adultes ou à des enfants? Il est connu que dans beaucoup de pays européens, le conte est devenu un genre enfantin par excellence, d'où viennent les changements au niveau de l'auditoire et de la manière de raconter le conte [Vlasova $1994: 8]$.

Dans le recueil de Cerquand, par exemple, il ne s'agit point de contes pour les enfants, parce qu'il a été publié dans le bulletin d'une société savante, tandis que les recueils de Barbier et de Webster pouvaient être utilisés pour les enfants, ainsi que pour les folkloristes, contenant des images et des commentaires, ainsi que la version basque. Juan San Martín écrit dans la préface de son recueil que celui-ci est conçu pour les enfants qui ont besoin de contes, mais parmi les recueils modernes, il en existe plusieurs dont les folkloristes et les enfants se servent.

\section{Le recueil de Vinson}

Pour beaucoup de recueils modernes de contes basques, surtout ceux qui ont été publiés en France, le livre de Vinson Le folk-lore du Pays basque [1883] est un point de repère. En plus des contes, le livre inclut des chansons, de différents dictons, proverbes, devinettes, etc. Dans son recueil, Vinson indique trois sources des contes : ceux que Webster et lui (en fait, c'était principalement Webster) ont collectés, des textes de Cerquand et un conte d'Araquistain [Vinson 1883 : xiv].

Dans son livre, Vinson a publié 35 contes dont 16 sont extraits des revues de Cerquand, 16 des cahiers de Webster, un du recueil d'Araquistain et encore deux ont été collectés en 1868 et 1881, probablement par Vinson lui-même : le conte collecté en 1881 est l'un des trois contes du manuscrit 720 de Vinson qui n’a pas été copié du manuscrit de Webster, de cette façon nous trouvons pour la première fois sa source basque.

Pour les contes de Cerquand, Vinson n'indique pas les instituteurs qui les ont collectés, et pour les contes de Webster il ne mentionne que le nom du conteur à la fin de chaque conte. Même en sachant le nom de famille, Vinson ne croit pas toujours nécessaire de le révéler : ainsi, il écrit Laurentine $\mathrm{X}^{* * *}$ dans son livre [Ibid. : 79], tandis qu'il sait (nous le voyons dans son manuscrit) que son nom de famille est Kopena [Vinson 1876 : 282].

Pour Vinson, le point principal dans la traduction est son caractère littéral. Dans la 
préface du recueil il écrit :

" J'ai traduit en effet, suivant mon habitude, aussi littéralement que possible [...]. Je n'ai jamais admis le système qui consiste à habiller d'un français académique et guidé des pensées exprimées dans les langues étrangères »; « Le lecteur sera peut-être aussi choqué de certains passages qui lui paraîtront trop libres. Je n'ai pas cru devoir les supprimer, parce qu'ils sont originaux et qu'ils donnent une idée plus complète de l'esprit basque »[Vinson 1883 : xxxiii, xxxiv].

Bien que les recueils de Vinson soient des compilations, il traduit les textes basques qui sont les meilleurs. Les textes français qu'il publie paraissent pour la première fois dans cette langue.

Bizarrement, pour les contes que nous trouvons dans le manuscrit de Webster, Vinson indique toujours l'année, bien que dans le manuscrit de Basque Legends, elle ne soit pas indiquée ni dans le livre, ni même dans le manuscrit de Vinson. Nous sommes amenés à nous demander s'il le fait au hasard (les années indiquées sont souvent 1874 ou 1875), ou si c'est Vinson qui a collecté ces contes ou qui participait à leur collecte.

\section{Le recueil de Carpenter}

Bien que tous les éditeurs contemporains choisissent des textes classiques pour leurs livres, leurs choix se distinguent dans une grande mesure. Par exemple, dans le livre Tales of Basque Grandmother de Frances Carpenter, on utilise plutôt les contes de Vinson, Webster et Barbier :

« Many [tales] are based on stories and myths recounted by M. Jules [sic !] Vinson in his "Le Folk-Lore du Pays Basque", by Rev. W. Webster in his treatise on "Basque Legends" and by those found in the pages of the Basque Magazine "Gure Herria". Several have for their basic source tales written by M. Emmanuel Souberbielle" » [Carpenter 1930 : 5].

Dans le recueil de Carpenter, nous trouvons beaucoup plus de digressions que dans les autres recueils postérieurs.

Nous citerons le début du conte du sabbat qui commence par la digression suivante:

«When Bidabe was a young man he decided it was a time to find himself a wife to help him tend his goats and his sheep and keep his soup pot fill and boiling. He looked about among the girls in his village, and one by one he passed the all except Kattalin and Iana-mayi. Between these two he found it hard to decide.

You see, Kattalin was the prettiest girl in the whole countryside ; but Ianamayi was the best of the bread makers. Kattalin's eyes were shining and black ; but Iana-mayi was hard-working and good. Kattalin could dance the fandango in a manner to delight the eyes of all beholders ; but Iana-mayi went every Sunday to church and everyone spoke well of her. 
However, after thinking and thinking, Bidabe decided he loved Kattalin best. He loved her bright smile and her soft rosy cheeks, and he liked the gay scarf she wore tired round her head. He would not believe certain old folk of the village who whispered behind their hands that Kattalin was a witch. Be she witch or be she fairy, he determined she was one he would marry.

One Saturday evening Bidabe went to pay a visit to the family of his brideto-be. He had planned as a surprise a pair of fine wooden shoes for his beloved Kattalin. He had cut two pieces of white cherry wood and modeled them to the shape of her dainty foot [...] » [Carpenter $1930: 25-26]$.

Même si les sources principales sont assez fiables par rapport aux autres (les textes de Webster et de Vinson), Carpenter arrange les contes à sa propre façon : en plus de changements considérables dans le style, l'adaptatrice donne des prénoms basques et non basques aux personnages alors que ces caractéristiques sont presque toujours absentes dans les textes originaux (Bidabe, Cattalin, Iana-mayi, Piarres, Ganiche, Gracianne, Chiko, Gachucha [Carpenter 1930 : 26, 82, 87, 143, 176, 191], Mayiana [Ibid. : 34] au lieu de Sabine [Souberbieille 2005 : 22] et d'autres).

Parfois le sujet du conte devient plus adapté aux oreilles des enfants. A titre d'exemple, nous pouvons comparer les deux versions du conte Le pou — celle de Vinson, la traduction du conte de Webster, et celle de Carpenter : dans cet épisode, il s'agit de la manière dont la mère du jeune homme vérifie qu'il s'agit d'une fille.

«La mère ne savait pas que penser et lui dit : « Moi, je lui demanderai de coucher avec moi, et alors je m'en assurerai. Si c'est une fille, vous vous marierez; si c'est un homme, je le ferai avec lui ». Cette dame dit donc au prince s'il voudrait coucher avec elle. Le prince lui dit que oui, certainement. Quand le soir est venu, ils vont au lit tous les deux. La dame lui touche les seins et les trouve durs, durs; elle allume de la lumière pour mieux s'en assurer, et voit que c'est véritablement une fille. Elle va dire à son fils que le prince sera pour lui s'il veut ; que c'est vraiment une fille, et bien charmante et bien faite » [Vinson $1883: 75-76]$.

«The next day, however, the Queen tip-tooed into Fifine's room when she was asleep. She found her dressed in a fine silken gown which she had put on for her afternoon's rest. Then at last she knew that her son's dreams were true, and that their guest was indeed a beautiful princess » [Carpenter $1930: 157$ ]. malade :

Carpenter substitue le motif de la lamina en couches par une lamina qui est

«Elles [la sage-femme et une lamina] entrent dans une grande chambre qui était la plus belle de toutes. Là, il y avait une Lamigna sur le point d'accoucher et en mal d'enfant; tout le tour de la chambre était garni de mignons petites êtres, tous assis et dont aucun ne bougeait jamais. Marguerite fit son office » [Vinson 1883: 41]. 
" Through one room, after another, each more splendid than the last, she was led on and on until she finally stepped into a great chamber, the most gorgeous of all. There on a bed in the center of the floor lay a pale beautiful lady who, she soon learned, was the Queen of the Fairies. Many fairy figures were seen in the vast room. They sat on the window sills, on the chairs and the couches, but the moved never a muscle. Without saying a word, they sat their bright eyes on Catiche as she began to care for the sick fairy. She bathed her and rubbed her and then she gave her a drink made of powerful herbs that quickly brought the roses back into her white cheeks. In a short time the fairy Queen sat up on her couch. Her ilness was gone» [Carpenter $1930: 241]$.

Les adaptations de Carpenter concernent souvent la fin des contes, par exemple, au cas où il s'agit du châtiment d'un imposteur ou si la fin est trop triste. Ainsi, dans le conte The Dragon with the seven heads on bat le charbonnier au lieu de le brûler [Ibid. : 195]. Dans le conte des trois vagues, l'adaptatrice supprime l'explication que les sorcières sont la femme et la fille du capitaine, donc le capitaine, au lieu de mourir de chagrin, se réjouit à cause de tous les poissons que son équipage a attrapés [Ibid. : 213].

Dans le conte des deux muletiers Carpenter enlève la fin triste. Nous citerons la fin de la traduction du texte de Cerquand faite par Vinson et l'arrangement de Carpenter. Il s'agit de la partie où les sorcières se doutent qu'il y a quelqu'un sous le pont qui aurait pu entendre leurs paroles:

« Elles y vont toutes et trouvent notre muletier, qui ne savait où se cacher. Lune le frappe, et l'autre le pousse. Après l'avoir ainsi ballotté, elles le jettent à l'eau, et là finit notre muletier trompeur. Lautre, au contraire, vécut riche et heureux au milieu de sa famille. Je vivais alors dans une petite maison, près de ce pont, et tous les soirs j'entendais les gémissements du muletier » [Vinson $1883: 19]$.

« All the witches trooped down under the bridge, and of course they found hiding there the Dishonest Mule Driver. He started to run away as fast as he could, but the caught him before he had gone more than two paces. This one beat him, and that one kicked him. Between them they finally tore off his clothes and threw him, bruised and beaten into the bramble bushes on the edge of the stream in the ravine. So you see in the end the Dishonest Mule Driver was very well paid for cheating his friend » [Carpenter $1930: 233-234]$.

Cependant, malgré les nombreuses digressions, Pierre Lafitte déclare la fidélité de Carpenter au folklore basque :

« Madame Carpentier [sic !] a reproduit fidèlement une trentaine de nos légendes : la simplicité du vocabulaire, la bonhomie de la syntaxe, la brève conclusion morale qu'Amatchi épingle sur chaque histoire, l'allure populaire du récit, rendue par quelques gentils américanismes piqués dans une belle prose anglaise [...]» [Lafitte 1931 : 83]. 


\section{Le recueil d'Anjou}

Pierre d'Anjou se veut conservateur des traîts originaux basques, bien qu'il se permette de nombreux changements en adaptant les contes:

« nous nous sommes refusés à faire ouvre d'adaptateurs, nous avons voulu conserver, autant que faire se peut, cette rudesse, mais aussi cette originalité qui donnera une idée - encore imparfaite — du goût et du génie de ceux qui conçurent cette œuvre, en rapport avec l'auditoire auquel elle était destinée » [Anjou 1946: 7].

Son recueil contient 22 contes. Dans le recueil d'Anjou, nous retrouvons 13 contes de Vinson (sept de Webster, cinq de Cerquand, un d'Araquistain), ainsi que sept contes de Barbier et deux contes traduits de Basque Legends de Webster [Webster 1877]. Nous pouvons constater que, contrairement au recueil de contes basques [CB 1978], les modifications concernent presque toujours les contes de Barbier.

Le système des notes utilisé par l'auteur nous paraît assez souvent insuffisant. Lauteur indique très rarement les sources de ses contes. Ainsi, d'Anjou crée une version littéraire du conte La nappe, l'âne et le bâton sans mentionner le recueil de Barbier d'où provient ce conte. Le premier conte, Le Pou, est tiré du recueil de Julien Vinson, Le folklore du Pays Basque (Vinson lui-même l'a repris des cahiers de Webster). Pourtant, d'Anjou ne mentionne ni l'un, ni l'autre. Malbrouc, La Tabatière, Le Fou et le Tartalo, La Mère et le Fils idiot, Le Triple Serpent, Les Trois Vagues, Les Deux Muletiers proviennent également du livre de Vinson que cite souvent Webster, que d'Anjou ne mentionne pas non plus.

Plusieurs contes ont été empruntés dans le recueil de Vinson qui a traduit les contes de Cerquand, pourtant d'Anjou n'indique que la note de Vinson : "Marie Oihenart, soixantedouze ans, de Bustance-Iriberry, Cerquand, 28 », « Jean Salaber d'Assurucq - Cerquand 52 » [Ibid. : 52, 84].

Très peu de contes sont empruntés à Webster (il s'agit de textes qui sont absents dans le recueil de Vinson).

D'un point de vue stylistique, quelques passages de retardement, typiques des contes merveilleux ${ }^{11}$, sont enlevés chez d'Anjou. Nous observons également dans le texte original l'emploi de deux temps, le présent et le passé, tandis que le conte d'Anjou emploie seulement le passé, selon l'usage littéraire. Bien que d'Anjou prétende ne pas vouloir faire d'adaptation littéraire, c'est précisément un travail d'adaptateur qu'il accomplit. Nous pouvons comparer le début des deux contes.

Le conte d'Anjou :

«Au temps où Seigneur Jésus accompagné de Pierre parcourait le pays des Basques, vivait dans une maison d'Ahusquy une vielle femme et ses trois fils.

11.- Par exemple, « Et il allait, allait toujours » chez Barbier. 
C'étaient de rudes et joyeux souletins aventureux et quelque peu contrebandiers. Un jour l'aîné dit à sa mère :

- Mère, faites-moi cuire des petits pains, ensuite j'irais de l'autre côté de la montagne faire fortune.

La mère fit ce que son aîné lui demandait. Quand les pains furent dorés à souhait le fils les mit dans la poche et s'en fut par monts et par vaux» [Ibid. : 17].

Le conte de Barbier :

« En d'autres temps, dans une maison, il y avait trois fils.

Un jour, l'aîné dit à sa mère : « Mère, faites vite les petits pains, que je m’en aille ensuite faire fortune. »

La mère fait les petits pains, et le fils s'en va par monts et pas vaux. » [Barbier $1931: 41]$

Nous ne pouvons pas douter qu'il s'agisse de deux variantes du même texte, les coïncidences étant trop remarquables. Il est également évident que le texte original appartient à Jean Barbier puisque la deuxième variante a été publiée plus tard. Les différences entre les deux textes sont dignes d'analyse, car le premier passage du texte que nous citons est deux fois plus long que le deuxième. Les différences entre les deux textes sont les suivantes.

Premièrement, les digressions de l'auteur servent à donner une couleur locale basque. On mentionne le nom d'une maison « dans une maison »- Barbier vs. « dans une maison d'Ahusquy »- d'Anjou ${ }^{12}$. On indique l'origine des jeunes gens — souletins, et leur caractéristique - «quelque peu contrebandiers », ce qui renforce l'image du Basque typique chez le lecteur. Si dans le conte de Barbier il s'agit d'un « jeune homme », Pierre d'Anjou parle du «Basque».

Deuxièmement, il y a dans le conte des digressions qui visent à le rendre plus littéraire : « le Seigneur Jésus lui donna une nappe » vs. « le Seigneur Jésus lui donna une nappe, une belle nappe blanche en fine toile », " sur cette nappe, aussitôt, tu auras tout le boire et manger qu'il faudra » vs. " aussitôt elle apparaîtra chargée de tout ce dont tu auras besoin pour boire et manger».

Si d'Anjou peut donner une explication au sujet des contes, il n'hésite pas à le faire. Ainsi, au lieu d'une courte phrase introduisant la parution du Seigneur Sauvage chez Barbier : «C'étaient des forêts effrayantes que les forêts de ces temps-là », il donne toute une description de la mythologie qui n'est d'ailleurs pas tout à fait basque « Mais, en ces temps-là, les forêts n'étaient pas comme aujourd'hui des lieux calmes et reposants où l'on vient se détendre et oublier les soucis de la vie. Bien au contraire, elles étaient le repaire de monstres, de lutins, de fées, d'animaux fabuleux, qui pouvaient à loisir perpétrer leurs forfaits ou dispenser leurs faveurs, suivant le tempérament de chacun d'eux ».

La demeure du Seigneur Sauvage devient « étrange et magnifique ». Au lieu du banal 
« Ils grimpent donc le long de la caverne » apparaît le texte romantique « Désireuse de retrouver le village, des êtres humains et le soleil, la mère consentit à suivre son fils ».

Pour d'Anjou, les valeurs traditionnelles basques sont également importantes : " Quelle joie de la pauvre mère » vs. «La pauvre mère pleurait, tant son bonheur était grand de revoir sa maison, son petit jardin et l'église où elle venait s'agenouiller si souvent » [Barbier 1931 : 84-85, Anjou 1946 : 107-109].

En adaptant les contes de Barbier, d'Anjou ajoute de petits passages moraux ${ }^{13}$ :

« Ayant reconnu la sainte chapelle, elle [la servante] se mit à crier : « Saint-Sauveur, faites-moi grâce, ayez pitié de moi ! »

Et à peine eut-elle achevé ces mots, qu'elle descendit à terre tout doucement, délivrée du mauvais esprit... » [Barbier 1931 : 81]

« La servante ayant reconnu la chapelle s'écria :

— Saint-Sauveur, faites-moi grâce ! Ayez pitié de moi !

Comme par enchantement, le vent qui l'entraînait dans les airs cessa de souffler, elle descendit à terre tout doucement, et délivrée du mauvais esprit, elle se promit que plus jamais elle ne serait cupide et coquette » [Anjou 1946: 126].

Dans le conte Le Fou et le Tartaro, d'Anjou omet un passage de Vinson, probablement par hasard — le même mot « anneau » est mentionné deux fois :

« La femme dit tout cela au garçon et lui donne les trois anneaux. Notre garçon sauve les trois malheureux en leur disant ce qu'ils avaient à faire.

Il arrive chez son maître et lui donne les trois anneaux un à un. Quand il prit le premier, il s'est mis à danser» [Anjou 1946 : 45 ; Vinson 1883 : 55].

D'Anjou n'ajoute pas d'épisodes supplémentaires ; tout ce qu'il change c'est la stylistique. Cela ressemble beaucoup aux modifications d'autres traducteurs et peut être expliqué par le fait qu'il est plus difficile de changer le contenu d'un conte que sa forme. A titre d'exemple, nous comparons encore deux extraits du conte de Barbier avec les modifications d'Anjou.

Et la reine riait !...

La version de Barbier :

« Dans une misérable chaumière vivaient trois frères. Ils connurent la promesse du roi et se dirent par devers eux-mêmes qu'il leur fallait bien voir si, de quelque façon, ils ne s'arracheraient pas un sourire à la malade.

Laîné prend donc avec lui un panier de pommes toutes rouges, et le voilà parti.

13.- Dans les versions de la même légende de Cerquand et de Webster [Cerquand 1882 : 259-260, Webster 1873 : 492] la jeune fille meurt. 
Tandis qu'il chemine ainsi, dans un trou à sorcières, et tombée là-dedans, il voit une vieille femme - une sorcière — qui ne réussissait pas sortir de là. La vieille se met donc à crier, pour lui demander un secours. Mais le jeune homme ne lui accorde pas un regard de plus et passe son chemin » [Barbier 1931: 96].

La version d'Anjou :

«Dans une misérable chaumière vivaient trois frères. Dès qu'ils eurent connaissance de la promesse du roi, ils se dirent qu'après tout ils pouvaient bien tenter leur chance, et qu'il leur fallait bien voir, si de quelque façon, ils n'arracheraient pas un sourire à la jeune reine.

Laîné partit le premier. Il emportait avec lui un panier de pommes rouges. Chemin faisant, il rencontra dans un trou à sorcières une vieille femme - une sorcière - qui tombée là, se débattait et criait à qui la sortirait.

Elle était laide comme un démon, et le jeune homme ne lui accorda qu'un regard. Il passa son chemin sans plus s'occuper d'elle et de ses cris ». [Anjou 1946 : 53]

\section{Le lac de Biarritz}

La version de Barbier :

«Et ces gens demeurèrent stupéfaits : ils voyaient la huche... bondé de pains jusqu'au bord...

Ils tombèrent à genoux devant le Seigneur Jésus. Et le Seigneur Jésus, alors, demanda s'ils pouvaient avoir un lit, car ils étaient bien fatigués, tous les deux » [Barbier 1931 : 59].

La version d'Anjou :

« Le gens demeurèrent stupéfaits. Leur surprise fut grande en constatant que la huche était bondée de pain jusqu'au bord.

Il en fut de même au charnier, qui vide l'instant d'avant se trouvait garni de salé. Dans le cellier le vin remplissait le tonneau qui n'avait jamais eu panse plus rebondie.

La femme leur prépara à souper.

Le Seigneur Jésus et saint Pierre après s'être restaurés se sentirent las.

Le Seigneur Jésus demanda:

— Avez-vous un lit à nous donner ?» [Anjou 1946 : 127].

De pareilles modifications concernent d'autres contes d'Anjou, empruntés à Barbier ${ }^{14}$, tels que Les lamignas du pont d'Ustalea. Dans le conte Le chandelier de Saint-Sauveur, d'Anjou 
modifie la version de Barbier, et non celles de Cerquand, plus anciennes [Cerquand 1874 : 279-280] :

«Quelques jours après, le valet fut de nouveau à la montagne ; il avait, la veille, battu le froment. Tout à coup, à un détour du chemin, se dresse le Seigneur Sauvage. Terrifié, persuadé que c'en est fait de lui cette fois, le valet se gratte la tête... Et voici que, entre ses doigts, se rencontrent trois ou quatre grains de blé, accrochés aux cheveux depuis la veille... Crac, il les porte à la bouche pour rompre le jeûne... et le Seigneur Sauvage disparu pour ne plus reparaître jamais. Mais jamais, non plus, le valet ne s'est aventuré à jeun dans la montagne » [Barbier 1931: 78].

« A quelque temps de là, le valet de Lahibarria retourna dans la montagne avec son troupeau. La veille il avait battu le blé qu'entre temps il allait faucher.

Tout à coup, au détour d'un sentier, le Seigneur Sauvage se dressa devant lui!

- Cette fois, je te tiens, clama le Seigneur Sauvage. Et terrifié, persuadé qu'en effet c'en était fait de lui, se gratta la tête.

Et voici que ses doigts rencontrèrent quelques grains de blé égarés dans sa chevelure depuis la veille.

Pieusement, il les porta à sa bouche pour rompre le jeûne.

Le Seigneur Sauvage poussa un cri terrible que transmit l'écho de la vallée ; cette fois encore le valet lui échappait et le Seigneur Sauvage disparut pour ne plus jamais reparaître car jamais plus le valet ne s'aventura à jeun dans la montagne» [Anjou 1946: 104].

\section{Le recueil de Reicher}

Dans le recueil de Gil Reicher, l'auteur n'indique que le lieu d'enregistrement du conte et ne dit rien des locuteurs et des autres sources des contes. Parfois, Reicher introduit des éléments novateurs qui s'écartent du folklore traditionnel. Ainsi, Tartalo chez Gil Reicher est chrétien. Dans la légende de Tartalo qui enlève une jeune fille et qui a un enfant d'elle ${ }^{15}$, c'est la jeune fille elle-même qui veut se marier avec Tartalo, séduite par ses richesses :

« [Tartalo :] Personne ne connaît le palais que j'habite. Celle qui y régnera sera la plus riche de la terre.

Alors, Margareta, la troisième fille d'Uberua, qui était coquette et ambitieuse, s'avança :

— Je veux bien t'épouser, Tartalo, si M. le Curé y consentent » [Reicher 1947 : 3].

15.- La légende qui est conservée dans beaucoup de recueils de contes du Pays basque et des territoires voisins. 
Les dernières paroles de Tartalo ( « Tu es la première femme qui ait bien voulu épouser un Tartalo, tu es la première à posséder ses richesses » [Ibid. : 4]) nous rappellent plutôt le Prince de La Belle et le Bête.

Dans les contes de Reicher, les laminas « ne sont pas méchants; ils ont besoin d'homme. Ils ne demandent qu'à l'aider et qu'à l'aimer » [Ibid. : 7]. Ayant étudié les autres textes folkloriques parlant des laminas, nous pouvons observer que cette image est trop idéaliste.

Les contes que Reicher emprunte aux recueils classiques sont modifiés. Pauvres laminak correspond au sujet d'une lamina en couches, qui est présent chez différents éditeurs ; Piarres et le vent du nord trouve ces racines dans La lune d'Azkue [Azkue 1942 : 408] ; Cattalina et Errege Salomon, une histoire fondée sur la légende du roi Salomon, est dérivée du conte de Cerquand [1875 : 183-185].

En changeant la version plus récente d'un conte de Barbier Le lac de Biarritz [Barbier 1931 : 58-59], Reicher transforme la légende étiologique du lac de Biarritz en déluge de Noé.

La version de Barbier :

«Cette nuit là il y eut d'innombrables éclairs, d'innombrables coups de tonnerre ; la pluie vint en trombe, le vent en tourbillon... Le lendemain, emportées par les eaux, toutes les maisons de voisinage avait disparu, et disparu également tous leurs habitants.

La maison qui avait recueilli le Seigneur Jésus et Saint Pierre fut la seule à demeurer debout...

S'il vous arrive d'aller jamais du côté de la Négresse, vous la verrez encore là-bas, au bord du grand lac créé par la pluie, durant cette nuit terrible » [Barbier 1931: 59].

La version de Reicher :

« La méchanceté, l’orgueil, la dureté des hommes ne faisaient que croître. Alors, dieux, pour les punir, envoya le déluge.

Les grandes eaux s'abattirent sur la terre, gonflant les torrents, faisant déborder les lacs, les vagues de la mer se soulevèrent et recouvrirent les côtes ; la pluie tomba sans arrêt pendant quarante jours et quarante nuits. Tout disparaissait sous les eaux, les maisons, les champs, les bois, tous les hommes périrent.

Mais dans un village du Pays basque, autour d'une pauvre maison, un rempart magique empêchait les eaux de tomber. La pluie s'arrêtait à l'orée du pré. Un toit d'eau recouvrait la demeure, mais ne crevait pas. A travers la pluie, les anges bâtissaient un escalier de cristal, sur lequel un jour descendit, resplendissant, Jésus qui venait rappeler sa promesse.

Les gens de la maison continuèrent à vivre, les blés du champ à pousser, le fournil à chauffer.

Un lac se forma au-dessus. Puis, la colombe apporta à Noé le rameau d'olivier et les terres réapparurent. Les collines séchèrent, les arbres repoussèrent, et la vie recommença jusqu'à ce que de nouveaux crimes des hommes viennent encore une fois attirer la colère divine. 
Sous le lac, tout est en paix.

Et vous pouvez y descendre, si vous trouvez la première marche de l'escalier» [Reicher $1947:$ 83-84].

\section{Le recueil de Thomasset}

Dans le recueil de René Thomasset, plusieurs contes qui se trouvent dans la partie intitulée Contes récents sont tirés du recueil de Jean Barbier (La haie de joncs, La reine de la forêt (= Une reine dans la forêt chez Barbier), La nappe, l'âne et le bâton). Pourtant, Thomasset ne donne aucune référence bibliographique. Il mentionne seulement que les deux derniers contes ont été traduits par P. Rectoran (« D’après une traduction de P. Rectoran ») [Thomasset 1962 : 118], mais il ne donne pas la source originale de ces contes.

Nous pouvons cependant remarquer que les textes dans les deux recueils sont assez différents d'un point de vue stylistique.

Même les formules folkloriques ( Il y avait, une fois, un homme et une femme ») sont éliminées et remplacées par une narration littéraire. Mais les changements les plus importants consistent à enrichir le vocabulaire du conte, à modifier légèrement sa grammaire et à expliquer les passages qui pourraient être mal compris par le lecteur : "Le roi mis en défiance » chez Barbier [1931 : 51] vs. «Le roi se méfia de cette nouvelle, car il avait remarqué avant son départ, l'hostilité que sa mère témoignait à sa femme » chez Thomasset [1962: 121]. Il s'agit d'une tendance générale de cette époque : s'il y a quelques omissions dans les textes originaux des contes, les auteurs des recueils modernes essayent de les compléter.

Les changements considérables sont plutôt rares. Nous pouvons néanmoins remarquer quelques modifications dans les contes : « le Seigneur Jésus avait baptisé les deux enfants de la reine, les appelant, l'un le Soleil, et l'autre la Lune » [Barbier 1931 : 52] vs. « Il baptisa ensuite lui-même les nouveau-nés, nommant l'un Chalvat (Sauvé) et l'autre Pierrech (Pierre) [Thomasset 1962 : 123]. Pourquoi fallait-il changer le nom des enfants ? Peut-être, ils étaient trop paiens pour un conte basque? Et le nom de Chalvat est une belle démonstration de l'importance de la foi catholique chez les Basques, tandis que le nom de Pierrech est un nom classique basque.

Dans le conte La nappe, l'âne et le bâton, René Thomasset insère des éléments de la couleur locale qui n'existaient pas dans le texte original de Barbier : «Un jour, l'aîné dit à sa mère » [Barbier 1931 : 46] vs. "Un jour, l'aîné dit à l'etcheko-anderea (maîtresse de la maison)» [Thomasset 1962 : 127] ; «-Aïché $\left.{ }^{1}\right)$ [Interjection basque] s'exclama l'homme, tout effaré, quelle aubaine! » [Ibid. : 129]. Sur la nappe merveilleuse apparaissent les plats basques et même bearnais («Quelle garbure! Quelle piperade ! [...] Quelle coque au lait ! (crème renversée) et quel Irouléguy » [Ibid. : 128] qui sont absents chez Barbier.

Dans un autre épisode, il est également possible de noter des changements. Dans le conte original de Barbier, la reine, Marie-Valentine, aperçoit un homme qui s'est enfoncé une épine dans la main et après l'avoir retiré, « elle s'aperçut alors que cet homme était son père !... » [Barbier 1931 : 54]. Dans le conte « dérivé », en apprenant que quelqu'un s'est enfoncé une épine dans la main, la reine "ne doute pas un instant qu'il s'agit de son père » [Thomasset 1962 : 126].

Peut-être est-ce destiné à rendre le conte encore plus merveilleux. 


\section{Les recueils de Bidart}

Beaucoup de compilateurs constituent leurs recueils à partir de ceux des premiers folkloristes, sans changer considérablement le texte. C'est le cas du recueil de Pierre Bidart. Le compilateur utilise une nouvelle orthographe : Haxko vs. Hachko chez Barbier. Le seul critère selon lequel Pierre Bidart choisit les contes pour son recueil est le critère géographique : il s'agit des contes de Basse-Navarre et de Labourd. Les deux livres contiennent beaucoup de photos du Pays basque, ce qui peut être intéressant d'un point de vue culturel.

Le titre des recueils de Bidart est trompeur. Ainsi, le premier volume de son ouvrage s’appelle Récits E Contes populaires du Pays Basque recueillis par Pierre Bidart en Basse Navarre, et le second Récits E Contes populaires du Pays Basque recueillis par Pierre Bidart dans le Labourd [Bidart 1977a, 1977b], ce qui donne l'impression que c'est Bidart lui-même qui les a collectés sur le terrain.

Lattribution des contes dans les recueils de Bidart est souvent erronée. Parmi les contes qui, selon Bidart, viennent du Labourd, nous en trouvons plusieurs qui ont été contés par les habitants des autres provinces du Pays basque de France.

Ainsi, le conte de Cerquand Le pêcheur et ses fils [Cerquand 1882 : 262-265], que le compilateur place parmi les contes labourdins, a en fait été récité par une personne originaire de Sainte-Engrâce, en Soule. Le conte de Cerquand, L'épouse à la recherche de son mari [Ibid. : 275-278], vient d'Arhansus en Basse-Navarre et non pas du Labourd. Le même défaut concerne les contes bas-navarrais contenant beaucoup de contes de Barbier. Mondarrain, où habitent les laminas [Barbier 1931 : 18], se trouve au Labourd. C'est un souletin qui aurait raconté à Barbier le conte Et la reine riait, placé dans le volume bas-navarrais [Barbier 1931 : 153]. Pour plusieurs contes de Barbier nous n'avons aucune donnée géographique, par conséquent leur attribution est complètement arbitraire. Le lecteur pourrait comprendre, pourquoi la légende des laminas de Saint-Pée se retrouve dans le volume labourdin, étant donné que Saint-Pée est un village labourdin. Mais la plupart des attributions sont plus difficiles à comprendre. Bien que Bidart écrive dans son tome des contes de la Basse-Navarre : «Labbé Jean Barbier [...] s'intéresse particulièrement aux contes et légendes de la Basse Navarre dont il est originaire » [Bidart 1977 : 21], Barbier lui-même n'écrit nulle part qu'il préfère cette province ; au contraire, il cite les trois provinces du Pays basque français (le Labourd, la Basse-Navarre et la Soule) comme la source de ses contes [Barbier $1931: 5]$.

Bidart indique régulièrement les recueils d'où les contes ont été tirés. La plupart d'entre eux viennent des recueils classiques, du recueil d'Ariztia et de la revue Gure Almanaka, mais il y en a trois racontés par U. Aranguiz et D. Xebero, probablement recueillis par Bidart lui-même.

\section{Le recueil de Cosem}

Michel Cosem « s'est imprégné » des recueils de Cerquand, Webster ${ }^{16}$, et Barbier, ainsi que du Dictionnaire illustré de la mythologie basque de José Miguel de Barandiarán [Ba- 
randiarán 1993] et de l'ouvrage Quelques éléments pour découvrir la mythologie basque ${ }^{17}$. La plupart des contes du recueil sont assez courts, certains (La jument blanche) étant raccourcis par Cosem.

Pour ajouter une touche de couleur locale dans ses textes, Cosem indique (parfois, assez arbitrairement) les noms des lieux où se déroule l'action, ou le lieu d'origine du héros, plutôt dans les parties introductives des textes : «Mahistruba n'avait pas de bateau et il en était désolé. Chaque matin, il allait sur les quais d'Hendaye regarder les vagues d'océan »; « Manex, un pauvre homme de Tardets, ne savait plus combien il avait d'enfants » [Cosem 2008: 11, $111]$.

Le recueil de Cosem, ainsi que plusieurs autres, est un exemple typique de la double modification du conte : d'abord celle du traducteur et ensuite celle de l'adaptateur. A titre d'exemple, nous citerons le conte La mère jalouse (La mère jalouse et la jeune persécutée), où l'un des instituteurs de Cerquand, et très probablement Cerquand lui-même, traduit le texte et où ensuite Cosem le modifie. De cette façon, il s'agit souvent de chaînes de transformations, qui ne sont pas très longues, car les compilateurs reprennent les recueils originaux.

La version originale basque de Cerquand avec notre traduction :

«La mère jalouse ${ }^{18}$

Baciren ama alhaba batzu biciqui propiac. Nescatoco haren amatchia, çoina baitcen sorguina, juan cen egun batez bere alhabatchiaren ikhustera. Haren amari eguiten dasco laudorioac ceren duen hain alhaba edera. Ama horec galdeguiten deyo heya hura baina ederrago denez, phena luquela hala baliz eta galarastea isseya litekela, sobera berantu gabe. Sorguinac erraiten dio segurqui alhaba bera baino propiago dela, et nahi badu, hura cargatcen dela galarasteaz. Ama horec ez du bertceric galdeguiten eta beraz igortcen du bere alhaba promenatcera araxaldi batez bere amatchirequin » [Cerquand $1882: 281$ ].

Il y avait une mère et une fille très belles. Un jour, la marraine ${ }^{19}$ de cette fille, qui était sorcière, alla voir sa filleule. Elle fait des compliments à sa mère, parce qu'elle a une si belle fille. Cette mère lui demande si elle [la fille] est plus belle qu'elle, qu'elle serait peinée si cela était et que si cela était, elle essayerait de la faire périr, sans trop tarder. La sorcière lui dit que bien sûr sa fille est plus belle qu'elle, et que si elle [la mère] le veut, elle [la sorcière] se chargera de sa perte. Cette mère ne demande rien d'autre et donc, un soir, elle envoie sa fille se promener avec sa marraine.

La traduction du recueil de Cerquand :

« La mère jalouse et la jeune persécutée

Une fille était si belle que sa mère en devint jalouse. La marraine de cette

17.- Nous n'avons pas pu retrouver cet ouvrage.

18.- Les titres dans les versions française et basque sont différents.

19.- « Amatchi » : grand-mère ou marraine. 
beauté va la voir un jour. C'était une sorcière qui ne se plaisait qu'au mal. Elle connaissait bien la jalousie de la mère, en espérant y trouver une occasion de nuire à sa filleule. Pour l'exciter encore, elle se répandit ce jour-là en longs compliments sur les perfections de la jeune fille, jusqu'à ce que la mère, n'y tenant plus, lui dit : " La vue de ma fille est un supplice pour moi. Si vous m'avez conservé quelque affection, vous m’en débarrasserez.

La méchante marraine n'attendit que cette parole. Elle prit sa filleule pour l'accompagner, disait-elle, pendant sa promenade... » [Ibid. : 211]

La deuxième version française est encore plus éloignée de l'originale que la première. La version de Cosem :

«Ce jour-là, la sorcière, voyant la mère d'Amélie, ne cessa de lui faire compliments sur sa fille. La mère, excédée, lui dit :

- Si vous avez quelque affection pour moi, vous m'en débarrasserez.

La méchante sorcière n'attendait que cette parole. Elle demanda à sa filleule de l'accompagner... » [Cosem 2008 : 95].

La traduction en français est mieux organisée et plus cohérente que le texte original. Dans la version de Cosem, apparaît un prénom qui est absent du texte basque, et qui, de plus, n'est pas du tout basque. Le texte adapté garde quelques expressions du texte traduit. La version de Cosem est la plus courte parmi les trois textes.

Les modifications de Cosem, notamment le raccourcissement des textes trop longs des contes merveilleux, aboutit à la perte de motifs essentiels, tels que le motif du marin épargnant la vie au serpent, qui joue un rôle important dans la thématique. A titre d'exemple nous citerons le texte de Basque Legends et celui de Cosem :

« LIKE many others in the world, there was a master mariner. Having had many losses and misfortunes in his life he no longer made any voyages, but every day went down to the seaside for amusement, and every day he met a large serpent, and every day he said to it :

"God has given thy life to thee ; live then.»

This master mariner lived upon what his wife and daughter earned by sewing. One day the serpent said to him :

«Go to such a shipbuilder's, and order a ship of so many tons burden. Ask the price of it, and then double the price they tell you » [Webster 1879: 100].

« Mahistruba n’avait pas de bateau et en était désolé. Chaque matin, il allait sur les quais d'Hendaye regarder les vagues de l'océan. Il s'asseyait sur les rochers et avait envie de pleurer.

Un jour, il rencontra un gros serpent qui parlait.

- Tu ne peux pas vivre ainsi, sans gagner le moindre sou, vivant du travail de ta femme et ta fille. Non, tu es un capitaine et un marin. Va voir le constructeur de navires que tu connais, commande-lui un beau bateau et paye-le le double du prix qu'il demandera $»$ [Cosem 2008: 11]. 


\section{Contes Populaires et Légendes du Pays basque}

Ce livre fait partie des recueils de contes traditionnels des régions différentes de France, et c'est la raison pour laquelle l'éditeur n'y utilise pas les contes d'Azkue et de Barandiarán.

Lattribution de certains contes est erronée. Ainsi, Le râteau qui est attribué à Cerquand, est en fait tiré du livre de Vinson et représente une traduction du conte de Webster, bien que des histoires similaires soient présentes dans le recueil de Cerquand. A la fin du conte, l'éditeur ajoute une remarque absente chez Vinson : "Cette maison de verre, on pouvait encore la voir, avec le corps de la jeune fille, au temps de la Restauration ». Nous trouvons encore une indication incorrecte dans la table des matières : il s'agit d'un conte de « W. Webster et J. Vinson » (Qu'est-ce que c'est que se marier) [Ibid. : 361], et en réalité, nous ne trouvons pas ce conte chez Webster.

Donc, compte tenu des indications erronées de l'éditeur, la plupart des contes a été tirée du recueil de Barbier (27) et de celui de Vinson (14 contes, dont neuf viennent de Webster, trois de Cerquand, un de Vinson même et un d'Araquistain). Le recueil contient également deux contes de Webster, évidemment, traduits par d'Anjou, qui n'est pas mentionné dans la bibliographie ${ }^{20}$. Nous trouvons dans le recueil deux contes de Cerquand et un conte de Carlos de Monts. D'autres textes (Møurs, croyances et superstitions) n'appartiennent pas au genre de contes.

La préface de ce recueil écrite par Mme Charles d'Abbadie d'Arrast mérite une attention particulière, car elle décrit une veillée dans la maison basque, tandis que la plupart des contes du livre n'ont pas été collectés pendant des veillées. Dans cette partie, on cite également le conte des mouches de Mendiondo tiré du recueil de Vinson, qui l'a emprunté chez Cerquand, ainsi que des contes de laminas et Basa-Jaun, un conte de la fournée d'Ahurhutze, une parabole de Jésus Christ et Saint-Pierre, un conte de deux bossus et quelques devinettes tirés de la même source [Vinson 1883].

Bizarrement, dans une remarque, l'auteur de la préface mentionne que Basa-Jaun est un diminutif d'Antoine, et non pas l'homme de forêt [CB 1978 : 12-27].

Dans les Contes Basques, les contes de Barbier sont mieux présentés, que ceux des autres folkloristes, bien qu'ils soient les moins originaux, ce recueil contient aussi des modifications légères : « Et il était riche ainsi et pour toujours » [Barbier 1931 : 50] vs. « Et il se trouva riche » [CB 1978 : 276]. Ce sont plutôt les versions de Barbier que Contes Basques donne presque sans modifications, bien qu'ils soient les plus littérarisés. Ainsi, parmi trois versions du Chandelier de Saint-Sauveur apparaissant chez Cerquand, une version chez Vinson ${ }^{21}$ et une version chez Barbier, l'éditeur choisit celle de Barbier, bien qu'évidemment elle soit secondaire par rapport à celle de Cerquand. Le texte d'autres folkloristes est reproduit assez fidèlement, mais certains contes sont abrégés. Le conte La trahison punie (Le traître puni) du recueil de Cerquand, qui a été modifié par le traducteur et l'éditeur, se trouve changé encore une fois.

20.- [Anjou 1946] et encore 2 contes de Webster.

21.- Traduction du manuscrit de Webster. 
« Bi muthil gazté chortian eroriac phartitu ciren soldado elgarrequi eta gorphuts bererat » [Cerquand $1882: 260$ ].

«S’il y a jamais eu deux amis inséparables, c'étaient Goyenetche et Etchegoyen, nés le même jour dans deux maisons voisins d'aspects également misérable. Eeur amitié datait du moment où its eurent la liberté de se rouler dans la poussière, sur la porte du logis paternel. Dès lors on les vit user leur eulottes sur les mênes banes à l'école et au catéehisme, mener leurs chèvres le long des mêmes haies et grandir jusqu'au moment où its furent appelés par la eonseription. Ils eurent la même chance de tirer un mauvais numéro et allèrent ensemble rejoindre le régiment $»$ [CB $1978: 45$, Cerquand 1882 : 148] ${ }^{22}$.

«Hurbildu cen erregueren palaciouri, eta nola aitcinachegui beyçouen, hartu çuan galdeguiten ceelaric eya nor cen eta han cer hari cen ? Eman ceen arrapostia nola yaquin çuen erregueren alhaba eri cela ; medicu cela eta haren sendorastera heldu cela. Berri horren entcutiarequi erreguec berehala sar eraci cuen medicu berri hura eta yuan eraci eriaren aintcinera » [Cerquand 1882 : 261].

« à la fin il arriva au palais du roi. Hallait entrer sans façon dans la cour lors= que les gardes l'arrêtèrent en lui demandant qui il était et ee qu'il venait faire.

"Je suis le doeteur Goyenetehe. J'ai entendu dire que la fille du roi est matade et je viens la guérir."

tes gardes allèrent avertit le roi d'Italie qu'il y avait un médecin à la porte du palais, qui venait pour guérir la prineesse. Le roi fit entrer le médecin chez lui et te condurisit dans la chambre de la malade. Puis it [Celui-ci dit] à Goyenetche: « Docteur, si vous parvenez à guérir ma fille, je vous donnerais autant d'argent que vous voulez et je ferai de vous mon gendre. » [CB 1978: 48, Cerquand $1882: 151]$.

Léditeur raccourcit également un texte de Vinson qui d'habitude est reproduit fidèlement :

Le recueil original de Vinson :

«Quand le père arrive, ce monsieur lui dit s'il veut lui donner sa fille pour femme. Le père lui répond s'il est venu là pour se moquer d'eux et si pour cela qu'il le fait arriver après avoir quitté son travail. Ce monsieur lui dit qu'il ne s'agit aucunement de moquerie, et que s'il veut lui donner sa fille pour femme, il lui donnera dans le pays une maison pour lui et tout ce dont il aura besoin pour manger et boire. Quand ce père eut entendu cela, il lui dit : « Oui, bien 
volontiers je vous donnerai ma fille », car il voyait qu'il avait besoin de bien passer le reste de sa vie, étant bien fatigué de vivre dans ces bois, et qu'il avait assez de peine à se sortir d'affaire. Son oeil s'était même rajeuni, et il était devenu tout joyeux de voir que la fortune de sa fille était faite et que lui-même allait être bien. Le père et la fille sont donc vite d'accord avec ce monsieur. Il leur donne une grande bource pleine d'or, (leur disant) d'attendre jusqu'à ce qu'il envoie d'autres nouvelles, et qu'il leur enverrait une voiture pour les chercher ; cette fille devait aussi s'acheter les vêtements nécessaires et prendre une fille de chambre pour arranger bien. Ces ordres donnés, le monsieur s'en revient à la maison, laissant le père et la fille fort contents » [Vinson 1883: 99].

Le recueil ultérieur :

«Quand le père arrive, il lui demande sa fille pour femme qu'il lui donnera une maison pour lui et tout ce dont il aura besoin pour manger et boire. Quand le père eut entendu cela, il lui dit : « Oui, bien volontiers je vous donne ma fille. » Le père et la fille sont donc vite d'accord avec ce prétendant qui leur donne une grande bource pleine d'or, leur disant d'attendre jusqu'à ce qu'il leur envoie une voiture pour les chercher. Cela dit, il revient chez lui, laissant le père et la fille fort contents » [CB 1978: 152].

Dans le recueil, sauf les textes qui sont raccourcis, l'éditeur ajoute quelques modifications : « il part, disant à son père et à sa mère qu'il va visiter les environs, et emportant avec lui beaucoup d'argent » [Vinson 1883 : 98] vs. " part, disant à son père et à sa mère qu'il va visiter les environs, et il emporte avec lui beaucoup d'argent afin de mieux plaire » [CB 1978 : 151]. Certaines corrections ne sont pas liées au sens, mais à la syntaxe du texte : "Malbrouc alla à la maison. Sa femme était une sorcière. Ils avaient trois filles. Le petit Malbrouc grandit beaucoup » [Vinson 1883: 80] vs. « Là, où il se trouvait, chez les sorciers qui avaient déjà trois filles, le petit Malbrouc grandit beaucoup » [CB 1978 : 74]. Dans le contes Les deux muletiers, l'éditeur des Contes Basques ajoute un mot : «tous les soirs j'entendais les gémissements du muletier fantôme » [Ibid. : 354].

Bien que la plupart des folkloristes modernes croient nécessaire de transcrire et d'éditer les textes le plus fidèlement possible, il en existe certains qui croient indispensable d'adapter le texte. Nous attestons cette attitude moderne par rapport au texte dans le conte La jument blanche: "Ce conte est reproduit ici tel qu'il était dit en 1875 par un conteur de Saint-Jeande-Luz. On pourra cependant reprocher au transcripteur de ne pas avoir «nettoyé » un peu mieux ce texte, sans doute par souci d'en garder le pittoresque » [Ibid. : 63]. La même remarque concerne le conte Malbrouk $k^{23}$, où l'éditeur ajoute plusieurs corrections. Ainsi, le processus de «nettoyage » des contes est considéré comme normal, voire nécessaire. En parlant du conte La mère et le fils idiot et Qu'est-ce que c'est que se marier [Ibid. : 145, 151], l'éditeur remarque :

23.- « Même note que pour le conte précédent » [Ibid. : 73]. 
«Conte transcrit tel qu'il a été dit par le conteur », comme une sorte d'excuse, ce qui reflète son avis négatif ${ }^{24}$.

D'autres recueils de contes basques et les contes basques dans d'autres recueils Les auteurs de plusieurs recueils utilisent les textes folkloriques comme matériaux didactiques. Ainsi, dans le livre de Beobide, le style du conte de Barandiarán Errana ta amagiarraba 'La belle-fille et la belle-mère' est complètement modifié. Nous pouvons noter les différences au niveau phonétique (Errena ta amagiñarreba dans le titre de Beobide). Les calques espagnols sont corrigées (« bajatu zan errekara »vs. « jaitsi zan errekara »elle descendit au ruisseau', « iru golpiek emon » vs. « iru kosk-kosk emon » 'frapper trois coups', " palazio eder bat » vs. « jauregi eder bat » 'un beau palais', « iru kazadore » vs. « iru eiztari » 'trois chasseurs', « kriaduak » vs. « morroiak » 'les domestiques', « eutzi trapue eskuek sikaketako » vs. « artu eskuak legortzeko zapia » 'prends la serviette pour essuyer les mains'). Les changements au niveau grammatical concernent la déclinaison biscayenne ( «alaba biyekin » vs. « alaba biakaz » 'avec les deux filles'), ce qui rend le texte plus cohérent, mais moins authentique.

Comme bien souvent dans les œuvres de vulgarisation, on ajoute les détails qui manquent à l'avis du compilateur du livre:

" gero umeak ure eskatu eutzan amari : ama, ure gure dut nik, ama »'ensuite les enfants ont demandé de l'eau à leur mère : mère, je veux de l'eau, mère' vs. «Basoan umeak egarritu, eta eske hasi ziran : Ura, ama, ura. Ura nai dot, ama... » 'Dans la forêt l'enfant a eu soif et ils ont commencé à demander : De l'eau, mère, de l'eau. Je veux de l'eau, mère';

« kriaduak kendu eutzesan esku biek erranari » 'les domestiques ont coupé les deux mains à la belle-fille' vs. " morroia'k, errukituta, beso biak bakarrik kendu eutzozan amari »'les domestiques, pris de pitié, n'ont coupé que les deux mains à la mère’ [Barandiarán et al. 1962 : 16-17 ; Beobide’tar 1980 : 84].

La même finalité didactique est soulignée dans le recueil d'Angel Irigarai qui utilise les contes de Barandiarán et Azkue:

« La finalidad que persigue la Academia de la lengua vasca al editar este libro es doble. Presentar por un lado Cuentos y Leyendas [... ] en lenguaje vivo y natural para solaz de los lectores vascos, niños y mayores ; y que sirva también para los escolares de esta idioma, gracias a la versión castellana y a las notas aclaratorias que van acompañados » [Irigarai $1957: 121$ ].

24.- De plus, le conte de Vinson [1883 : 98-102], comme nous l'avons déjà vu, est considérablement raccourci. 
Léditeur évite d'être trop littéraire et renonce à utiliser une transcription phonétique fidèle qui prendrait en compte toutes les particularités des parlers locaux. Angel Irigarai ne change pas le texte espagnol de Barandiarán.

En ce qui concerne le texte basque, presque tous les changements sont d'ordre phonétique. Si Barandiarán a publié un texte très proche de la transcription phonétique, cette dernière est remplacée dans le recueil d'Irigarai par une notation orthographique (« bê »=> « bere » 'son', « ementzôn » => « omen zuan » 'il avait (paraît-il)', « oiakôn »=> « oerakoan » 'en se couchant', « ei'zak » => « egin zak »'fais-le', « beiz ê » => « berriz ere »'de nouveau'). Léditeur ne change jamais le lexique des contes, en revanche, au niveau syntaxique, il modifie légèrement l'ordre des mots : « eta motille oso alai bê etxea jûn ementzan » => « eta mutilla joan zan oso alai bere etxera » 'et il paraît que le jeune homme s'en alla chez lui très joyeux' [Ibid.].

En analysant les recueils modernes, il faut également mentionner un livre publié par C. Clavería Arza qui ne décrit pas toute la richesse du folklore basque. Il cite quelques contes et légendes et les analyse, y compris les personnages, ne donnant les sources des contes que très rarement. Certains contes ont des similitudes avec ceux des recueils classiques, mais le compilateur n'indique pas quelles variantes ont été utilisées [Clavería Arza 1958].

Dans les recueils modernes, nous pouvons remarquer des problèmes de traduction. Ainsi dans un conte, le traducteur traduit l'expression « belle fille» par un terme de parenté :

« Une veille de la St-Jean, à l'aube, une belle fille entra chez la maîtresse de la maison Gorritépé. "Bonjour, Marguerite... » vs. " Al término de una noche de San Juan, Margarita, la nuera de la señora de la casa Gorritepe, regresaba a la hora en que el sol está a punto de salir » [Cerquand 1875 : 214, MLV 1986 : 49].

Le plus souvent, dans la plupart des éditions de vulgarisation le pourcentage des contes merveilleux est très faible. Cela concerne surtout les éditions peu volumineuses. Nous pouvons supposer que pour les éditeurs les contes merveilleux semblent les moins authentiques, tels ceux que l'on peut en trouver dans n'importe quel livre de contes. Ainsi, dans le recueil de René Thomasset [Thomasset 1962], il n'y a qu'un seul conte merveilleux. Pour lui, les contes les plus typiques du Pays basque sont les récits de corsaires, de joueurs de pelote et de contrebandiers. Dans le recueil de Gil Reicher [Reicher 1947] on peut également trouver quelques récits de personnages historiques (Dechepare, Iparagirre), mais les contes merveilleux sont absents. Nous voyons bien que les éditeurs de contes choisissent soit beaucoup de contes merveilleux, soit des récits parmi lesquels aucun ne relève du genre.

Aujourd'hui nous pouvons trouver plusieurs recueils de contes basques sur internet. Ainsi, les contes de Webster sont présentés en anglais (le site http://www.sacred-texts.com/ neu/basque/bl/index.htm correspond à [Webster 1879]).

Les mêmes contes, en basque, de Webster se trouvent sur le portail Klasikoen Gordailua : http://klasikoak.armiarma.com/idazlanak/W/Webster.htm correspondant à [Webster 1993]. Le recueil de Jean Barbier [1929] également : http://klasikoak.armiarma.com/ idazlanak/B/BarbierM ixterio.htm, ainsi que le recueil d'Errose Bustintza, Euskalerriko ipuñak 1950-1952 : http://klasikoak.armiarma.com/idazlanak/B/BustintzaEIpunak.htm.

Les contes collectés par Cerquand sont accessibles sur le portail gallica (gallica.bnf.fr), 
dans la version fac-similé du Bulletin.

Plusieurs contes basques traduits en russe et adaptés à partir de la version française avec de nombreuses modifications se trouvent sur un portail des contes du monde http://fairytales.su/narodnye/baskskie-skazki.

Parfois les contes basques sont utilisés dans des recueils parmi d'autres contes de France. Ainsi, dans l'anthologie des contes de France, où Dévigne inclut les contes de Picardie, Angoumois, Basse Bretagne, Champagne, Corse, Lorraine, Touraine, Pays basque, etc. et qu'il appelle un livre « où les Français de toutes nos provinces retrouvent assemblés en un monument expressif, les vieux récits et les traditions populaires de leur pays » [Dévigne 1950: 13], l'éditeur ajoute un conte basque. Parmi les nombreux textes basques, il choisit seulement le conte Les trois vagues du recueil de Vinson Le folklore du Pays Basque [Vinson 1883], qui est lui-même une réédition d'un texte d'Araquistain [1866]. Il abrège et modifie considérablement le texte. Ainsi, dans la variante ultérieure de la légende des trois vagues, manque l'épisode qui explique que les sorcières sont la femme et la fille du capitaine.

Michel Cosem, pour son recueil Contes traditionnels des Pyrénées [Cosem 1991], ne choisit que deux contes venant du Pays basque : La trahison punie de Cerquand et Les sept voleurs de Barbier, versions du recueil [CB 1978].

Les seuls recueils que choisit l'éditeur d'un autre livre, Contes merveilleux des pays de France [CMF 1991], parmi les recueils classiques des contes basques, sont l'édition anglaise des contes de Webster, dont il traduit The Grateful Tartaro et Heren-Suge [Webster 1879 : 22-32], et l'édition de Barbier (L'agneau noir, une traduction du conte Et la reine riait !) [Barbier 1931 : 96-99], les types courants en Europe.

\section{Conclusion}

Pour conclure, nous pouvons dire que dans les œuvres de vulgarisation utilisant les contes basques, la structure du conte n'est jamais altérée, aussi bizarre et confuse soit-elle (c'est le cas des contes composés, comme Malbrouk, Le pêcheur et ses fils [Webster 1873 : 465-479, Cerquand 1882 : 262-265]). En revanche, on essaie de « basquiser » le conte au niveau phonétique et syntaxique (on corrige les calques), aussi bien qu'au niveau stylistique.

Au début du XXI ${ }^{\text {ème }}$ siècle on commence à rééditer les textes classiques de contes basques $^{25}$. Nous pouvons supposer que maintenant que les contes basques faisant partie du manuscrit de Webster de Basque legends sont traduits en français, et que plusieurs contes de Cerquand le sont en espagnol, ces textes seront utilisés par des compilateurs pour créer de nouveaux recueils de contes basques. 


\section{Bibliographie}

Anjou, Pierre d'. Contes populaires du Pays des Basques. Collection « Contes de Prêt ». Paris : Le Liserou. 1946.

Araquistain, Juan V., Tradiciones vasco-cantabras. Tolosa : Impr. de la provincia, 1866.

AzkUe, Resurrección María de. Aintziñako ipuiñak. Zarautz: Itxaropena, 1968.

AzkUE, Resurrección María de. Euskalerriaren Yakintza. Literatura popular del País Vasco. II: Ipuin eta irakurgaiak, Madrid: Espasa-Calpe, 1942.

Barandiarán, José Miguel de. Dictionnaire de mythologie basque. Donostia : Elkar 1993.

Barandiarán, José Miguel de, y colaboradores. El mundo en la mente popular vasca. Tomos I-IV. San Sebastián : Auñamendi, 1960-1962.

BARBIER, Jean. Ichtorio-Michterio. Bayonne: Impr. du « Courrier », 1929.

Barbier, Jean. Légendes basques. Paris : Delagrave, 1931.

BEobide'TAR, José Luis. Euskalerriko ipuiñak. Bilbao : Euskerazaleak, Asociación para el fomento del vascuence. 1980.

BIDART, Pierre. Récits et contes populaires du Pays Basque/2 recueillis par Pierre BIDART dans le Labourd. Paris : Gallimard, 1977a.

BidART, Pierre. Récits et contes populaires du Pays Basque/1 recueillis par Pierre BIDART en BasseNavarre. Paris : Gallimard, 1977b.

Carpenter, Frances. Tales of Basque Grandmother. New York: Doubleday, Doran and Company Inc., 1930.

Catálogo = Catálogo tipológico de los Cuentos Populares Maravillosos Vascos. Herri kulturaren narrazioak, 2007.

$C B=$ Contes Populaires et Légendes du PAYS BASQUE. Richesses du Folklore de France. Paris : Les Presses de la Renaissance. 1978.

CerquAnd, Jean François. Ipar Euskal Herriko Legenda eta Ipuinak. Anuntxi Aranaren Transkripzioa. Donostia: Txertoa, 1986.

Cerquand, Jean-François. Ipar Euskal Herriko Legenda eta Ipuinak. Anuntxi Aranaren Transkripzioa. Ipuinak, Fabulak, Parabolak. Donostia: Txertoa, 1985.

Cerquand, Jean-François. Légendes E Récits Populaires du Pays basque // Bulletin de la Société de sciences, lettres et arts de Pau. 1874-1875. II série. Tome $4^{\text {ème }}$, pp. 233-289 ; 18751876. II série. Tome $5^{\text {ème }}$, pp. 183-260 ; 1876-1877. II série. Tome 6 ème, pp. 450-531 ; 1882-1883. II série. Tome 11 ${ }^{\text {ème }}$, pp. 101-294.

Cerquand, Jean-François. Légendes \& récits populaires du Pays Basque. Anglet : Aubéron, 2006. 
Clavería Arza, Carlos. Leyendas de Vasconia. Pamplona: Gómez, 1958.

CMF = Contes merveilleux des pays de France. Franchesse : Iona, 1991.

Cosem, Michel (Sélection et réécriture). Contes du Pays Basque. Toulouse : Milan, 2008.

Cosem, Michel. Contes traditionnels des Pyrénées. Toulouse : Milan, 1991.

DÉvigne, Roger. Le légendaire des provinces françaises à travers notre folklore. Paris : Horizons de France. 1950.

EHK = Euskal Heriko Kondariak. Joan B. Arakistainek bere "Tradiciones vasco-cantabras" liburuan jasotakoak. Donostia: Elkar-Debako kultur elkartea, 1986.

EtXaniz ErLe, Xabier (-ren edizioa). Geure ipuinak. Iruña : Pamiela, 2000.

Gorostidi Manuel. Leyendas vascongadas. Euskara, 1878.

IRIGARAI, Angel. Euskalerriko ipuiñak = Cuentos populares vascos: con su versión castellana . Zatautz: Itxaropena, 1957.

Lafitte, Pierre. Folk-Lore. Labbé J. Barbier et Mme Frances Carpentier [sic !] // Gure Herria. Janvier-Février 1931, pp. 82-85.

$M L V=$ Mitos y leyendas vascos. Prologo y épilogo de Andrés Ortiz-Osés. Madrid: Zero, 1986. Reicher, Gil G. Récits et Légendes Basques. Bordeaux; Paris : Bière, 1947.

Souberbieille, Emmanuel. En Pays Basque. Contes et rêveries. La Rochelle: La Découvrance, 2005 ( $1^{\text {ere }}$ edition 1921).

Thomasset, René. Contes et Légendes du Pays Basque. Paris : Fernand Nathan, 1962.

VInson, Julien. Basque Legends. Manuscrit. (MS 720, Bibliothèque Municipale de Bayonne), 1876-1878.

Vinson, Julien. Le folklore du Pays Basque. Paris : G.-P. Maisonneuve \& Larose, 1883.

Webster, Wentworth. Basque Legends. Manuscrit. (MS 88, Bibliothèque Municipale de Bayonne), 1873-1875.

Webster, Wentworth. Basque Legends: Collected, Chiefly in the Labourd, by Rev. Wentworth Webster, M. A., Oxon. With an Essay on the Basque Language, by M. Julien Vinson, of the Revue de Linguistique, Paris. London: Griffith and Farran, 1877.

Webster, Wentworth. Basque Legends: Collected, Chiefly in the Labourd, by Rev. Wentworth Webster, M. A., Oxon. With an Essay on the Basque Language, by M. Julien Vinson, of the Revue de Linguistique, Paris, together with Appendix: Basque Poetry London: Griffith and Farran, 1879. (http://www.sacred-texts.com/neu/basque/bl/ blo0.htm).

Webster, Wentworth. Ipuinak. Xipri Arbelbideren edizioa. Donostia: Klasikoak, 1993.WebsTER, Wentworth. Légendes basques. Recueillis principalement dans la province du Labourd. 
Anglet : Aubéron. 2005.

ВлАсовА, Галина Ивановна. Методика собирания сказок в современности // Методические указания по собиранию фольклора. Москва : ГРЦРФ, 1994, pp. 6-15. (Vlasova, Galina I. La méthodologie de la collecte des contes contempotaines).

http://fairy-tales.su/narodnye/baskskie-skazki

http://gallica.bnf.fr/

http://klasikoak.armiarma.com/idazlanak/B/BarbierMixterio.htm

http://klasikoak.armiarma.com/idazlanak/B/BustintzaEIpunak.htm

http://klasikoak.armiarma.com/idazlanak/W/Webster.htm 